\title{
The effect of supervised and home based exercises on balance in elderly subjects: a randomized controlled trial to prevent falls
}

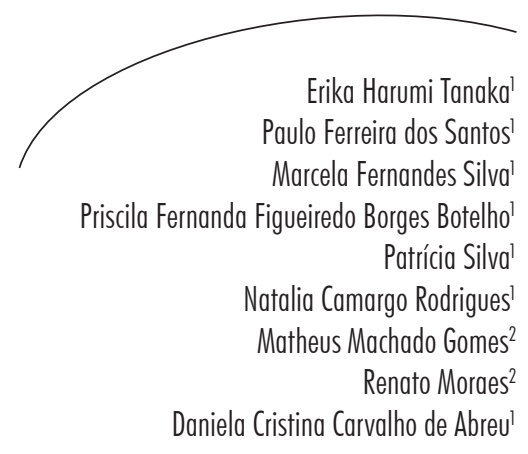

Abstract

Objective: The aim of the present study was to evaluate the influence of a balance training program on the semi-static balance of elderly persons by comparing a supervised group with individual home-based application. Method: A blinded randomized controlled multiarm trial was conducted. The elderly individuals were randomized into: Supervised Group (SG; $n=18)$; Domiciliary Group (DG; n=20) and Control Group (CG; n=18). The SG and DG participated in twice weekly training sessions for 10 weeks. A posturography evaluation was performed based on velocity, anterior-posterior (AP) and medial-lateral (ML) medial amplitude variables in firm surface with eyes open (FSEO) and closed (FSEC), tandem stance with eyes open (Tandem EO) and closed (Tandem EC), and single-leg stance (SL) situations. Two-way ANOVA and Tukey's post-hoc were used for parametric data, the Friedman and Wilcoxon post-hoc tests were used for intragroup analysis and the Kruskal-Wallis and Mann-Whitney post-hoc tests were used for intergroup analysis. Results: In intergroup analysis, the DG group showed improvement in body sway in the Tandem EC (velocity and medial amplitude AP) and single-leg stance (medial amplitude ML) situations. The SG showed a decline in the Tandem EO situation in all the variables. In intergroup analysis, the DG showed improvement in the FSEO position (medial amplitude ML), in the Tandem EC position (medial velocity ML), and the single-leg stance position (medial amplitude AP and ML). The SG showed improvement in the FSEO position (medial amplitude ML) and the single-leg stance position (medial amplitude AP), but showed a decline in the FSEO (variable medial velocity AP) and Tandem EO position (medial amplitude AP). Conclusion: The exercises were beneficial for the balance of the elderly individuals, with the DG presenting the best results. REBEC: RBR-3S9M65.

\footnotetext{
Universidade de São Paulo, Faculdade de Medicina de Ribeirão Preto, Departamento de Biomecânica, Medicina e Reabilitação do Aparelho Locomotor, Curso de Fisioterapia. Ribeirão Preto, São Paulo, Brasil.

2 Universidade de São Paulo, Escola de Educação Física e Esporte de Ribeirão Preto. Ribeirão Preto, São Paulo, Brasil.
}

Research funding: Fundação de Amparo à Pesquisa do Estado de São Paulo (Research Support Foundation of the State of São Paulo) (FAPESP), process nº 2012/05666-4, through Master Degree scholarship award.

Correspondence

Erika Harumi Tanaka

E-mail: erika.tanaka@gmail.com

Key words: Physical Therapy Specialty; Elderly; Postural Balance, Exercise. 


\section{INTRODUCTION}

Postural control, which is considered good when postural sway is low, 1 is affected by the aging process. Increased postural sway can be verified by an increase in the amplitude of center of pressure (COP) fluctuations. ${ }^{2}$

Several studies have identified the benefits of exercise on the overall health of the elderly, ${ }^{3-5}$ notably in the prevention of falls, ${ }^{6,7}$ and a lack of postural control may be corrected through exercise-based intervention. ${ }^{8}$

A number of studies have shown that combined training (multifactorial or multiple-factor) has greater benefits in preventing falls than training in isolation. ${ }^{8}$ Although some studies in scientific literature have evaluated the effect of balance training in groups $s^{2,9,10}$ and others have analyzed the effect of home-based balance exercises among the elderly, ${ }^{6,11}$ there is little research comparing group and home-based balance training in the same study. ${ }^{12}$ No study has been found that predominantly considers balance exercises applied through individual home-based and supervised group training to identify the most effective strategy for the implementation of exercises.

It is therefore important to study the effects of a training program based predominantly on balance exercises, in order to assess whether specific exercise programs to improve balance as a preventative measure have better results in the shortest period of time possible. According to the fall prevention guides of the American Geriatrics Society and the British Geriatrics Society, ${ }^{13}$ positive results have been achieved from training periods longer than 12 weeks, with sessions one to three times per week. The present study sought to evaluate whether ten weeks of training was sufficient to improve the balance of this population, when the treatment program prioritizes balance exercises.

In addition to reducing training time, a comparison of the same exercises performed in supervised groups or as individual homebased programs allow a wider range of options to be offered to elderly individuals who have difficulties attending therapy, considering that social factors (such as the encouragement, or the lack of encouragement, of other participants in the group, in other words the social awkwardness of performing a group activity); physical limitations (comorbidities, fear of falling when performing the exercise individually); competing priorities (little time to exercise due to family or work responsibilities); accessibility (lack of access to transport to therapy) and lack of motivation are barriers to adherence to therapy. ${ }^{14}$

The hypothesis of the present study is that ten weeks of predominantly balance based training, either unsupervised at home or in supervised groups, is sufficient to improve the postural control of elderly individuals, in order to offer this population a variety of health promotion options (either in groups in rehabilitation centers or at home alone) in order to improve adherence to fall prevention programs.

The objective of this randomized clinical trial was to evaluate the effect of a training program with predominantly balance exercises on the semistatic balance of the elderly, comparing supervised group and individual domiciliary application techniques.

\section{METHOD}

Design and ethical aspects of the study

A randomized controlled multi-armed clinical trial, registered with the Registro Brasileiro de Ensaios Clínicos - REBEC (the Brazilian Registry of Clinical Trials) in November 2011 under No. RBR-3S9M65, was performed. This study followed the recommendations of the Consolidated Standards of Reporting Trials (CONSORT) ${ }^{15}$ and was approved by the Research Ethics Committee of the Hospital das Clínicas of the Faculdade de 
Medicina de Ribeirão Preto of the Universidade de São Paulo (the Clinical Hospital of the Ribeirão Preto Medical School of the University of São Paulo) (FMRP/USP), under protocol number $5372 / 2010$. All participants in the study signed a free and informed consent form. The study was developed in the city of Ribeirão Preto, São Paulo, Brazil. The survey was conducted between the years 2011 and 2014.

\section{Recruitment/Selection}

The elderly persons who participated voluntarily in the study were recruited through leaflets which provided the phone number of the Laboratório de Avaliação e Reabilitação do Equilíbrio - LARE (the Laboratory of the Evaluation and Rehabilitation of Balance), the location of the assessments, and by spoken invitation. After interest had been expressed, the first contact with the elderly person was made by telephone, to identify if he or she met the study eligibility criteria.

The inclusion criteria were: elderly persons aged over 60 years, from the local community, of both genders, who were functionally independent. The participants had to be independently mobile, be normotensive or have blood pressure controlled by drugs. Type 2 diabetes mellitus was permitted, provided that the individuals were capable of detecting the application of at least $10 \mathrm{~g}$ on the soles of the feet using Semmes-Weinstein monofilament testing (SORRI ${ }^{\circledR}$, Bauru, SP, Brazil), according to the criteria adopted by the American Diabetes Association. ${ }^{16}$

The exclusion criteria were: participants with cardiovascular, neurological or musculoskeletal disease (with sequela or an impairment that interfered with semi-static balance); vestibular disorders; postural hypotension; foot deformities; visual disorders or cognitive deficit assessed by the Mini Mental State Examination (MMSE) ${ }^{17,18}$ Elderly persons who practiced Tai Chi Chuan or muscle strengthening exercises in the gym were also excluded, as muscle-strengthening also contributes to the improvement of postural control. ${ }^{8}$

To calculate the sample size the anteroposterior (AP) velocity of the COP variable (considered to be the main outcome of the study) was considered, taken as the mean and standard deviation values of a study of elderly persons aged over 60 from the community of San Carlos, Sao Paulo, ${ }^{19}$, taking into account a minimum alteration of $0.17 \mathrm{~cm} / \mathrm{s}$ in AP velocity (corresponding to $30 \%$ of the post-training improvement), resulting in a sample size $=12$ (per group), power $=0.8$, error $\alpha=0.05$.

Studies have suggested that average velocity is one of the most consistent and responsive postural control measures. ${ }^{20,21}$ The primary outcome was assessed at two time points (baseline and posttraining) along with the other Posturography variables - mediolateral (ML) velocity and mean $\mathrm{AP}$ and ML amplitude, which are considered as secondary outcomes in this study.

\section{Randomization}

Randomization was performed by a researcher who was not involved in recruitment or data collection and so had no direct contact with the research participants.

After the initial evaluation, simple randomization was performed using opaque envelopes from which three groups of options were randomly selected: the Supervised Group (SG), the Domiciliary Group (DG) and the Control Group (CG). The participants were not blinded. The evaluator performed two evaluations (baseline and after 10 weeks) and only after revaluations did the researcher responsible for randomization inform the evaluator about the groups to which the elderly persons had been allocated, so that data analysis could be carried out. 
Evaluation

Data collection in the present study was performed by blinded examiners (physiotherapists), who were trained for one year in order to learn how to use the assessment instruments.

All samples were collected from the LARE of the Department of Biomechanics, Medicine and Rehabilitation of the Locomotive System of FMRP/USP. Participants began by visiting the LARE to measure body mass and height.

Data regarding education, number of comorbidities and medications used were obtained through questionnaires structured by the authors, which included questions about demographic and socio-educational data. The level of physical activity and routine activities at home (10 questions) were obtained by the Baecke questionnaire modified for the elderly. ${ }^{22}$ This questionnaire was used as an exclusion criterion for patients who performed physical activities (gym or Tai Chi Chuan), and was also used during the revaluation process in order to identify whether an elderly person had altered their routine activities or the practice of sports during the study.

The MMSE was applied considering an educational level of 1 to 4 years, with the cutoff level set at 25 points. ${ }^{17,18}$

Semi-static balance was evaluated using a force platform in two phases: initial and posttraining. A follow-up was not carried out. Five different conditions were adopted: standing on a fixed platform with eyes open (FPEO) and closed (FPEC); barefoot and with feet spread to the width of the shoulders and with arms alongside the body (bipedal positions); in a tandem position with the right leg in front of the left with eyes open (Tandem EO) and closed (Tandem EC) and on one leg only (Unipedal) with eyes open.
Participants were instructed to remain as still as possible. The order of evaluation of each position was randomized and two analyzes were performed for each position. In eyes open situations, the elderly persons were told to look at a black circle, $5 \mathrm{~cm}$ in diameter, that was set in the wall in front of them at eye level at a distance of 1.5 meters. Elderly people who regularly used corrective lenses used these devices during the test, as visual acuity can interfere with postural stability. ${ }^{23}$

Body balance was evaluated using a force platform (EMG System do Brasil ${ }^{\circledR}$ ) with an acquisition frequency of $100 \mathrm{~Hz}$, which measured the distribution of the vertical load at four points, allowing analysis of semi-static equilibrium, with quantitation of amplitude and mean AP and ML COP displacement velocity. The COP signal was filtered by a fourth order Butterworth low pass filter with $10 \mathrm{~Hz}$ cutoff frequency. The displacement and trajectory of the COP in AP and ML directions was analyzed using the Matlab program (Math Works, Inc.). The average oscillation amplitude was calculated by subtracting the mean value of a time series for each point and obtaining the standard deviation of said time series. The average amplitude corresponds to the variance of the COP values and was used to estimate the stability of the COP. The average velocity of the COP was calculated by dividing the total displacement in each direction by the total collection period. The mean error of the Root Mean Square (RMS) for the actual localization of the COP on the force platform was $0.02 \mathrm{~cm}$, according to factory calibration.

Intervention

The intervention was performed for the SG and the DG twice a week in 55 minute sessions for a total of 10 weeks.

The SG performed 20 sessions. These sessions were in groups with a maximum of six elderly 
persons, supervised by two physiotherapists (with at least two years' experience in the area of geriatric physical therapy and the group rehabilitation of patients) who were not the evaluators, with blinding maintained. However, the therapists were aware that the elderly persons were part of a supervised training group. The exercise program was conducted at the Centro Integrado de Reabilitação (the Center for Integrated Rehabilitation) (CIR) linked to the Hospital Estadual de Ribeirão Preto Center (the State Hospital of Ribeirão Preto).

The DG had two supervised intervention sessions in the CIR to learn the exercises which they then performed in 18 individual homebased (unsupervised) activity sessions. These were performed twice a week for 10 weeks. To standardize and facilitate the exercises, all DG members received a DVD and an illustrative booklet demonstrating the exercises that should be done at home, and an activity form to be filled out by each individual after completion of the program, describing the day and time they performed the exercise, if all the stages were completed. Telephone contact was made with the participants of the DG each week to resolve any doubts about the exercises, and encourage them to carry out the activities.

The CG did not participate in the 10-week exercise program and did not receive any intervention in this period, with the data from this group being used for comparison. Whether or not the group maintained the same lifestyle habits during the 10 weeks was verified by structured questionnaires before and after the research period. Participants who changed their routine activities, as verified by the questionnaires, or performed some physical activity during the research period, were excluded from the study. For ethical reasons, following the research period and revaluation, individuals from the CG who were interested in participating in the exercise program performed the intervention program, either supervised or at home, according to their preference. The exercise program carried out by the SG and the DG comprised:

- Warm-up (5 minutes), repeat each movement ten times: alternate abduction, adduction, flexion and extension movements of the upper limb; alternate triple flexion movement of the lower limbs.

- Stretching (10 minutes) in three series, with an initial duration of 10 seconds for each series, increasing from week 4 to a duration of 20 seconds and from week 7 to 30 seconds: trunk and abdominal; pectorals, with extension of the upper limbs; glutes; quadriceps; hamstring; tibialis anterior; triceps sural.

- Semi-static and dynamic balance (35 minutes). Training was divided into exercises with the individual sitting and standing for 30 minutes, increasing to 35 minutes, in three series, with an initial duration of 30 seconds, increasing from week 4 to 60 seconds and from week 7 to 90 seconds.

- In the sitting position, the training involved transferring body weight to the right and left legs, at increasing speeds, moving from a support to a no support stance; rotating the torso with increasing speed; alternately lifting the legs from the ground; moving from a sitting position to a standing position, reducing the support base.

- In a standing position, training consisted of walking in a straight line (on firm ground and unstable ground, using mats): with a neutral head position (looking at the horizon); a moving head position (lateral rotation); with dissociation between the arm and the leg; standing, reducing the support base and transferring the weight to the right and left legs; standing and moving the body 
backwards and forwards; standing on one leg; walking on tiptoe; walking on heels; walking in zigzag; walking sideways; passing over obstacles; walking in tandem (one foot in front of the other); walking backwards; walking in curves and rotations; walking combined with a cognitive task; walking associated with a motor task (for example, transferring a ball from one hand to the other and passing a ball around the body in a circular motion).

- Cool-down (5 minutes): participants remain seated listening to their own choice of music, preferably soothing melodies.

Participants who performed at least $75.0 \%$ of the training sessions were reevaluated, ${ }^{24,25}$ using the intention to treat method.

\section{Statistical analysis}

Statistical analysis was performed using the intention to treat principle of analysis with mean imputation for COP analyses. By this principle, the result the elderly persons would have obtained if they had continued the training protocol was estimated. ${ }^{26,27}$

The Shapiro-Wilk test was used to analyze the normality of the anthropometric and COP data. The parametric data was analyzed using twoway ANOVA followed by Tukey post-hoc. Nonparametric data was analyzed by the Friedman test followed by the post-hoc Wilcoxon test to evaluate the paired data (intragroup analysis), and the Kruskal-Wallis test followed by the post-hoc Mann-Whitney test for unpaired data (intergroup analysis). All statistical analyzes were performed using SPSS software version 13.0 and the significance level was $5 \%(p=0.05)$.

\section{RESULTS}

Of the 186 elderly persons contacted by telephone, 130 refused to participate in the study or were excluded due to the eligibility criteria (nine were excluded for attending the gym). In total, therefore, 56 elderly patients were randomized into the three research groups (Figure 1). No elderly individuals were excluded at the time of reevaluation for changing their lifestyle during the research, as evaluated by the Baecke questionnaire. ${ }^{22}$

The acceptance rate for participation in the study was $37.63 \%$, representing the elderly who were contacted and agreed to participate in the balance training program for the prevention of falls. The adhesion rate (elderly persons who began the program and remained for its 10 -week duration) of the elderly persons in their respective groups was $72.22 \%$ for the SG; $60.0 \%$ for the DG and $72.22 \%$ for the CG.

During the training, 18 elderly persons $(\mathrm{SG}=5$, $D G=8$ and $C G=5$ ), who initiated the exercise protocol dropped out of the study. However, as the study was based on the intention to treat method, this data was included in the statistical analysis.- 


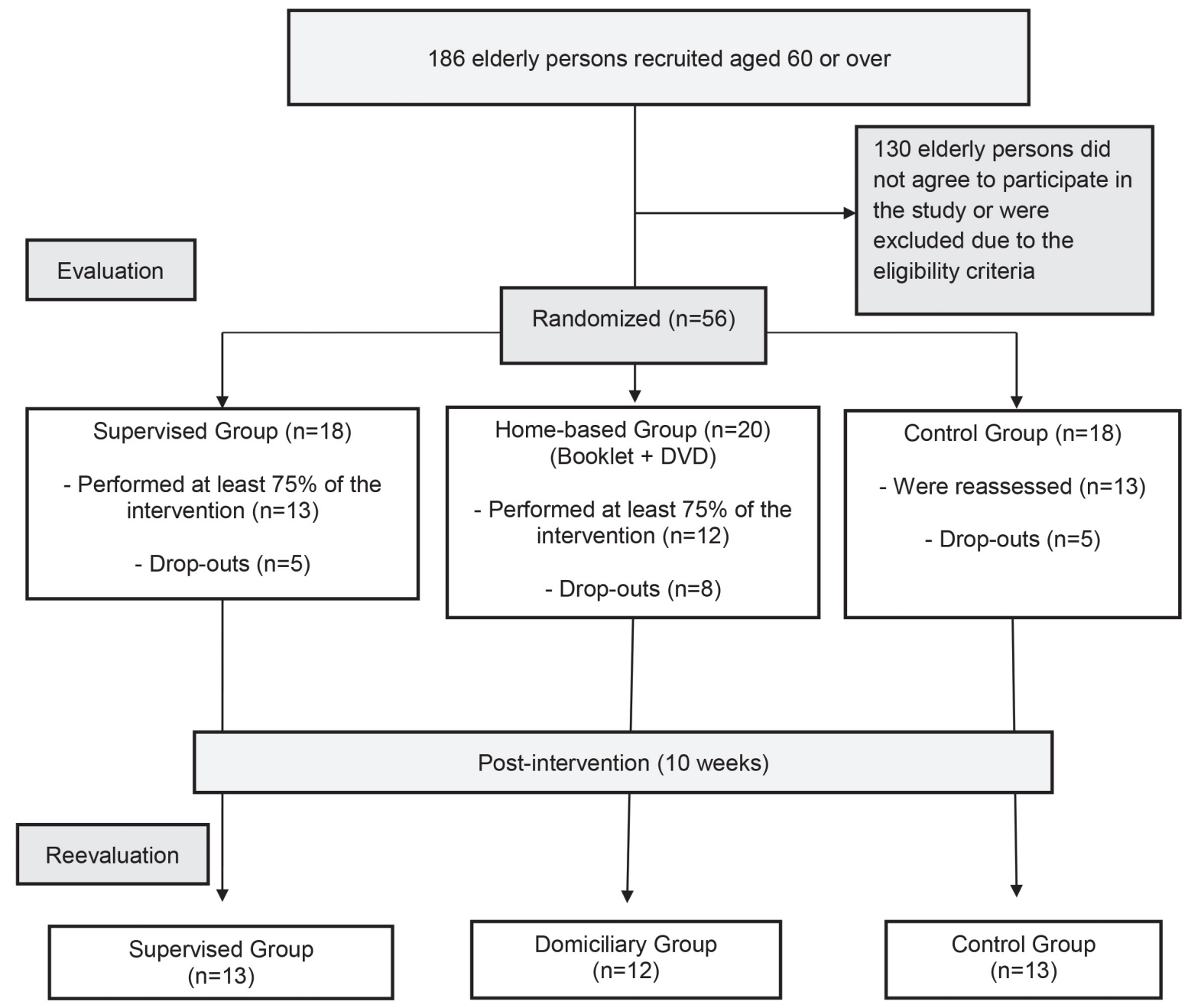

Figure 1. Flowchart of study participants. Ribeirão Preto, São Paulo, 2011-2014.

The SG therefore comprised 18 elderly persons, 13 of whom who completed the reevaluation and five who discontinued the intervention protocol. The DG contained 20 elderly persons, 12 of whom completed the reevaluation and eight of whom discontinued the intervention, and the control group contained 18 patients, 13 of whom who completed the reevaluation and five who declined to be reevaluated.

The reasons for withdrawal from the SG training were: transportation problems $(n=1)$, personal health problems $(\mathrm{n}=1)$, health problems of spouse $(n=1)$, competing priorities $(n=2)$. In the DG, the reasons for withdrawal were personal health problems $(n=3)$, health problems of spouse $(\mathrm{n}=1)$, competing priorities $(\mathrm{n}=4)$. In the control group, all claimed competing priorities $(n=5)$.

The sociodemographic characteristics (Table 1) were compared to verify the homogeneity between groups. The Kruskal-Wallis test revealed no differences in these variables between the SG, DG and CG. 
Table 1. Sociodemographic characteristics of sample. Ribeirão Preto, São Paulo, 2011-2014.

\begin{tabular}{lccccc}
\hline \multirow{2}{*}{\multicolumn{1}{c}{ Variables }} & \multicolumn{5}{c}{ Groups } \\
\cline { 2 - 5 } & Supervised & Domiciliary & Control & Total & p valor \\
Number of participants (\%) & $18(32.1)$ & $20(35.7)$ & $18(32.1)$ & $56(100)$ & -- \\
Women, n (\%) & $17(94.4)$ & $19(95.0)$ & $16(88.8)$ & $52(92.8)$ & -- \\
Age (in years)* & $66.4( \pm 3.5)$ & $65.7( \pm 5.4)$ & $66.7( \pm 4.1)$ & $66.2( \pm 4.4)$ & 0.47 \\
Height (in meters)* & $1.5( \pm 0.07)$ & $1.5( \pm 0.07)$ & $1.5( \pm 0.1)$ & $1.5( \pm 0.08)$ & 0.89 \\
Weight (in kilos)* & $66.1( \pm 15.5)$ & $68.2( \pm 9.0)$ & $72.3( \pm 18.9)$ & $69.0( \pm 14.9)$ & 0.71 \\
BMI* & $27.1( \pm 5.4)$ & $27.7( \pm 3.2)$ & $28.7( \pm 5.3)$ & $27.8( \pm 4.6)$ & 0.89 \\
Right side dominance, n (\%) & $16(88.8)$ & $19(95.0)$ & $17(94.4)$ & $52(92.8)$ & -- \\
Comorbidities* & $2.0( \pm 1.2)$ & $2.0( \pm 1.4)$ & $1.5( \pm 0.8)$ & $1.8( \pm 1.2)$ & 0.52 \\
Number of medications* & $2.6( \pm 1.6)$ & $2.2( \pm 1.8)$ & $2.1( \pm 1.6)$ & $2.3( \pm 1.7)$ & 0.65 \\
MMSE* & $28.5( \pm 1.7)$ & $28.4( \pm 1.7)$ & $27.69( \pm 2.0)$ & $28.2( \pm 1.8)$ & 0.32 \\
\hline
\end{tabular}

*mean and standard deviation; BMI $=$ body mass index $\left(\right.$ weight $/$ height $\left.{ }^{2}\right) ; \mathrm{MMSE}=$ mini-mental state examination.

In intragroup analysis, the CG showed an increase in average ML COP velocity in the FPEO, FPEC and Tandem EO positions. In the SG there was an increase in the average AP and ML velocity and mean AP and ML amplitude in the Tandem $\mathrm{EO}$ position. The DG presented a reduction in mean AP velocity and amplitude in the Tandem EC position and mean ML amplitude in the Unipedal stance (Table 2).

Table 2 also shows the Intergroup analyzes for all the conditions and variables. In the initial evaluation, differences were observed between the groups in relation to the conditions and variables analyzed, except for the Tandem EO and Unipedal positions. However, these initial differences were not sustained after treatment due to increased deterioration in the CG or an improvement in the intervention groups, suggesting that the training protocol improved balance in both groups (supervised and domiciliary). The only variable that maintained a difference the intergroup analysis after 10 weeks was AP amplitude in the Tandem EC position.

The DG showed improvement in average ML amplitude in the FPEO position, average ML velocity in the Tandem EC position and an improvement in the average AP and ML amplitude in the Unipedal position. The SG showed improvement in average ML amplitude in the FPEO position and mean AP amplitude in the Unipedal position, but a decline in mean AP velocity in the FPEO position and mean AP amplitude in the Tandem EO position. 


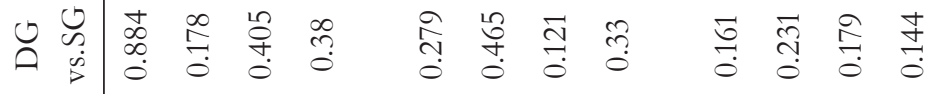
㩊

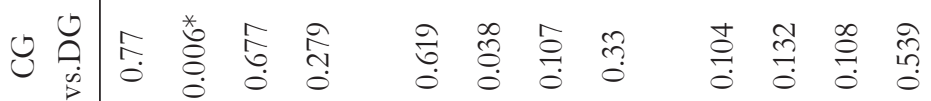

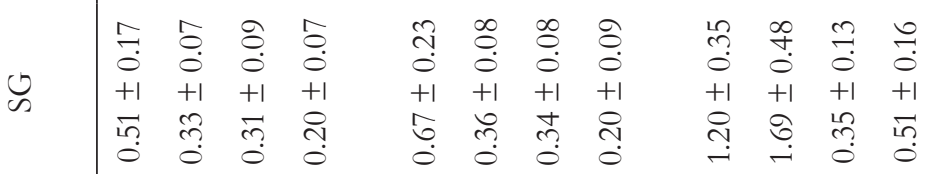




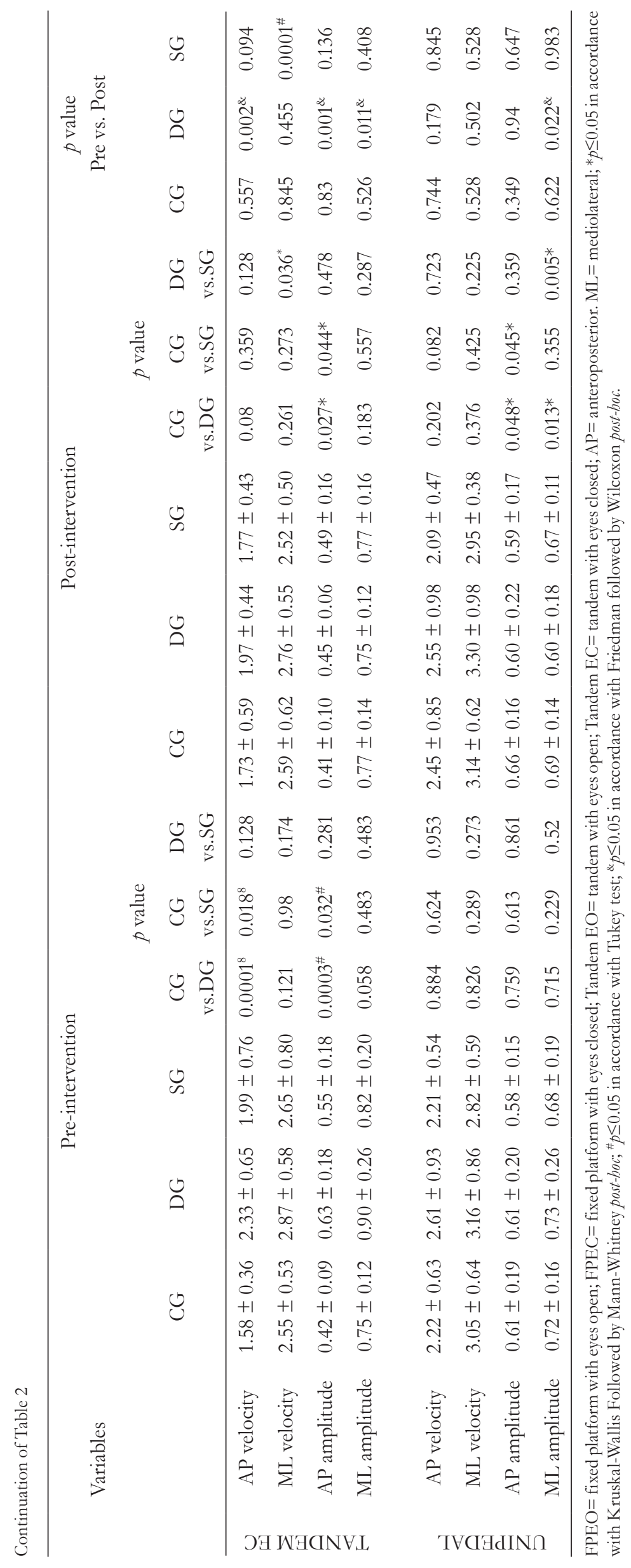




\section{DISCUSSION}

A number of studies have identified the benefits of physical exercise on the general health of the elderly. ${ }^{28,29}$ In terms of the prevention of falls in the elderly, there is scientific evidence through randomized controlled trials, systematic reviews and meta-analyzes that regular strength and balance training among this population can reduce the risk of falls by $15-50 \%,{ }^{5,30}$ However, the intervention based studies use various protocols and multicomponents, and few have assessed the effects of predominantly balance based training focused on preventing falls.

Additionally, based on practical experience of the low adherence of elderly persons to preventive exercise, it is important to compare the results of exercise programs applied in a supervised manner and those that are performed individually at home, expanding and adapting therapeutic strategy options for the needs and interests of the elderly.

Furthermore, predominantly balance-based training aims to focus on a specific component, which could allow therapeutic goals to be achieved in a shorter time, facilitating the return of individuals to routine habits and social and leisure activities that are often avoided due to postural instability and the risk of falls.

The results of the present research partly confirm the initial study hypothesis, as intergroup results suggest that both the SG and the DG achieved improvements in balance due to the exercise program. However, the domiciliary group tended to achieve greater benefits than the supervised group. In addition, the benefits were different for each group depending on the position adopted.

Studies have demonstrated the benefits of balance training in an orthostatic position on a force platform. ${ }^{2,31,32}$ Brouwer et al. ${ }^{31}$ observed significant improvement in the balance (AP and ML direction) of elderly patients following the completion of supervised (one hour per week) and home-based (40 minutes, twice a week) exercise programs, for eight weeks, involving low resistance exercises against gravity, using an elastic band for the legs and torso and reach, weight displacement and walking on the spot exercises. Also, Jessup et al. ${ }^{32}$ found a reduction in the mean total AP and ML oscillation in bipedal and tandem positions in a group of elderly women after 32 weeks of an exercise protocol that included stretching, warmup, muscle strengthening and balance exercises (tandem forward and backward walking, walking with obstacles) and going up and down stairs using vests of progressive weight. Penzer et al. ${ }^{2}$ observed a significant improvement in the balance of older people (average and maximum AP amplitude) in the bipedal position on foam after the completion of a supervised group exercise program (one hour, twice a week) for six weeks involving 10 minutes of warm-up and strengthening (three muscle strengthening exercises involving the lower limbs performed using equipment, strengthening the ankle extensors with an elastic band) or balance exercises (maintain balance on different surfaces: stiff, foam, bosu; with the eyes open and closed, and in bipedal, single leg and tandem positions), finalized by stretching.

In the present study intergroup analysis revealed a decrease in mean ML amplitude in the FPEO position for the DG, along with a reduction in mean ML velocity in the Tandem EC position and a decrease in mean AP and ML amplitude in a unipedal stance. The SG, however, displayed a reduction in mean ML amplitude in the FPEO position and a decrease in mean AP amplitude in a single leg stance.

In contrast, other training protocols did not achieve a reduction in the rate of falls or improved semi-static balance among the elderly. ${ }^{33,34}$ Lord et al. ${ }^{33}$ found no improvements in semi-static balance after an intervention program with individualized exercises that consisted of a 5 to 10 minute warm up, 30 minutes of group conditioning (strengthening, 
flexibility, coordination and balance exercises) and 10 minutes of individualized exercises (based on needs identified in evaluations), performed twice a week over 12 months. Another group received a minimal intervention through instruction sheets for exercises to perform at home, according to needs detected in an evaluation. The control group performed its habitual activities only. Ramsbottom et al. ${ }^{34}$ also failed to improve semistatic balance through a multicomponent training protocol performed twice weekly for 24 weeks. The hypothesis for the lack of improvement in semi-static balance after the completion of these training ${ }^{33,34}$ protocols included insufficient intensity, exercises that were either unsuitable for the population studied (considering that individuals with different functional skills trained in the same group) or non-specific (exercises focused more on the dynamic aspects of balance at the expense of semi-static balance). The training protocols that were not successful were supervised studies without specific training, ${ }^{8,34}$ those that used balance assessment positions that lacked sensitivity, such as a broad force platform base ${ }^{35}$, or those performed with insufficient intensity. ${ }^{33}$ According to Penzer et al., ${ }^{2}$ the contrasting findings stem from the variations in training regimens (intensity, duration, frequency) and balance evaluations (position of the feet, duration in the study protocols) used.

In the intra-group analysis of the present study, the SG displayed increased oscillation in the Tandem EO position (in both mean AP and ML amplitude and speeds), which may be associated with the fact that the exercise protocol was not specific to the individual needs of each participant, as individuals with different functional abilities were included in the same training group. In group training, the evolution of the exercises took place in a similar manner for all individuals, without considering the specific needs of each participant.

In the DG, despite the fact that this group followed an instruction booklet and DVD, the elderly individuals had the possibility of performing a specific type of exercise which presented difficulty for a longer period of time according to their individual needs, and could perform a more advanced exercise program twice a week. The freedom to design their own protocols may explain the more obvious improvements observed in this group. In contrast, safer exercises could be carried out for longer at the expense of more unstable exercises which are most challenging for balance, as each elderly person was responsible for his or her training, making them active agents in the promotion of their own health.

In the exercise protocol proposed in this study, the training time ( 10 weeks) was lower than the time recommended by the prevention of falls guidelines of the American Geriatrics Society and the British Geriatrics Society, which recommend 12 weeks $^{13}$, and few exercises were applied aimed at semi-static balance training (the principle of specificity). The exercises involving the orthostatic position were the single leg stance (which achieved an improvement post-intervention) and dynamic balance exercises.

Preventive action to improve semi-static balance, in relation to COP oscillation parameters, can help prevent the occurrence of falls in the elderly, as studies have shown that the risk of recurrent falls increases after an individual's first fall. ${ }^{4}$ However, further studies are needed to investigate exercise protocols that more effectively improve postural control, as well as more motivating and appropriate therapeutic strategies and instructions on the benefits of physical exercise for improving balance in older people, in order to achieve greater adherence to physical interventions. Additional studies for the creation of exercise protocols for preventing falls with increased progression and safely that can be applied both at home and in a supervised manner are important for clinical practice.

The present study had some limitations: the sample size and the non-inclusion of elderly persons from all age groups (the mean age of this population was 65-66 years) which limits extrapolating the results to the entire elderly population; the lack of a prediction for the dropout rate when determining the sample size; the subtle progression and the 
low difficulty of the procedures (as the exercises were designed to be safely performed by the participants at home without supervision); the lack of comparison of individual training with and without supervision; the short duration of the training (to avoid losses/dropouts) and the lack of analysis of whether the improvement was due to the number of repetitions performed during the week or the total duration of 10 weeks; and the lack of control of the performance of the home exercises, as the participants did not complete the daily exercise record, and no follow-up was performed.

\section{CONCLUSION}

The exercise protocol was sufficient to increase semi-static balance in both individual home-based and supervised group therapy strategies. Although the home group presented lower adherence to the program, these individuals obtained more benefits by reducing body sway on the fixed platform with eyes open (FPEO), tandem with eyes closed (Tandem EC) and single leg positions. The supervised group had lower body sway in the tandem eyes closed (Tandem EC) and unipedal positions, but showed deterioration in the fixed platform with eyes open (FPEO) and tandem with eyes open (Tandem EO) positions.

\section{ACKNOWLEDGEMENTS}

The authors would like to thank the Fundação de Apoio à Pesquisa do Estado de São Paulo (the Research Support Foundation of the State of São Paulo) (FAPESP) for funding and the Laboratory of the Evaluation and Rehabilitation of Balance of the Department of Biomechanics, Medicine and Rehabilitation of the Locomotive System of the Ribeirão Preto Medical School of the Universidade de São Paulo (the University of Sao Paulo) for providing the facilities to carry out this study.

\section{REFERENCES}

1. Strang AJ, Haworth J, Hieronymus M, Walsh M, Smart LJ Jr. Structural changes in postural sway lend insight into effects of balance training, vision, and support surface on postural control in a healthy population. Eur J Appl Physiol 2011;111(7):1485-95.

2. Penzer F, Duchateau J, Baudry S. Effects of short-term training combining strength and balance exercises on maximal strength and upright standing steadiness in elderly adults. Exp Gerontol 2015;61:38-46.

3. Reed-Jones RJ, Dorgo S, Hitchings MK, Bader JO. Vision and agility training in community dwelling older adults: incorporating visual training into programs for fall prevention. Gait Posture 2012;35(4):585-9.

4. Tanvi B, Feng Y, Yi-Chung P. Learning to resist gait-slip falls: long-term retention in communitydwelling older adults. Arch Phys Med Rehabil 2012; 93(4):557-64.

5. Uusi-Rasi K, Kannus P, Karinkanta S, Pasanen M, Patil R, Lamberg-Allardt C, et al. Study protocol for prevention of falls: a randomized controlled trial of effects of vitamin $\mathrm{D}$ and exercise on falls prevention. BMC Geriatr 2012;12:1-7.
6. Delbaere K, Valenzuela T, Woodbury A, Davies T, Yeong J, Steffens D, et al. Evaluating the effectiveness of a home-based exercise programme delivered through a tablet computer for preventing falls in older community-dwelling people over 2 years: study protocol for the Standing Tall randomised controlled trial. BMJ Open 2015;5(10):1-9

7. Gillespie LD, Robertson MC, Gillespie WJ, Sherrington C, Gates S, Clemson LM, et al. Interventions for preventing falls in older people living in the community. Cochrane Database Syst Rev 2012;9:1-5.

8. Alfieri FM, Riberto M, Gatz LS, Ribeiro CPC, Battistella LR. Use of clinical tests for verification of postural control in healthy elderly submitted to physical exercise programs. Acta Fisiátrica 2010;17(4):153-8

9. Ansai JH, Aurichio TR, Goncalves R, Rebelatto JR. Effects of two physical exercise protocols on physical performance related to falls in the oldest old: A randomized controlled trial. Geriatr Gerontol Int 2015. Epub Apr 14 
10. Alfieri FM, Riberto M, Abril-Carreres A, BoldoAlcaine M, Rusca-Castellet E, Garreta-Figuera R, et al. Effectiveness of an exercise program on postural control in frail older adults. Clin Interv Aging 2012;7:593-8.

11. Liu-Ambrose T, Davis JC, Hsu CL, Gomez C, Vertes $\mathrm{K}$, Marra C, et al. Action seniors! -Ssecondary falls prevention in community-dwelling senior fallers: study protocol for a randomized controlled trial. Trials 2015;16:144.

12. El-Khoury F, Cassou B, Latouche A, Aegerter P, Charles MA, Dargent-Molina P. Effectiveness of two year balance training programme on prevention of fall induced injuries in at risk women aged 75-85 living in community: Ossebo randomised controlled trial. BMJ 2015;351:1-10.

13. Summary of the Updated American Geriatrics Society/British Geriatrics Society clinical practice guideline for prevention of falls in older persons. J Am Geriatr Soc 2011;59(1):148-57. Developed by the Panel on Prevention of Falls in Older Persons, American Geriatrics Society and British Geriatrics Society.

14. Franco MR, Tong A, Howard K, Sherrington C, Ferreira PH, Pinto RZ, et al. Older people's perspectives on participation in physical activity: a systematic review and thematic synthesis of qualitative literature. Br J Sports Med 2015;49(19):1268-76.

15. Martins J, Sousa LM, Oliveira AS. Recomendações do enunciado CONSORT para o relato de estudos clínicos controlados e randomizados. Medicina (Ribeirão Preto) 2009;42(1):9-21.

16. Silva P, Botelho PFFB, D Guirro ECO, Vaz MM, De Abreu DC. Long-term benefits of somatosensory training to improve balance of elderly with diabetes mellitus. J Bodyw Mov Ther 2015;19(3):453-7.

17. Brucki SM, Nitrini R, Caramelli P, Bertolucci $\mathrm{PH}$, Okamoto IH. [Suggestions for utilization of the mini-mental state examination in Brazil]. Arq Neuropsiquiatr 2003;61(3B):777-81.Português.

18. Folstein MF, Folstein SE, McHugh PR. "Mini-mental state". A practical method for grading the cognitive state of patients for the clinician. J Psychiatr Res 1975;12(3):189-98.

19. Aveiro MC. Efeitos de um treinamento físico sobre o equilíbrio estático e dinâmico de mulheres idosas residentes na área de abrangência do Programa Saúde da Família de São Carlos [tese]. São Carlos: Universidade Federal de São Carlos; 2010.
20. Caballero C, Barbado D, Moreno FJ. What COP and Kinematic parameters better characterize postural control in standing balance tasks? J Mot Behav 2015;47(6):550-62.

21. Moghadam M, Ashayeri H, Salavati M, Sarafzadeh J, Taghipoor KD, Saeedi A, et al. Reliability of center of pressure measures of postural stability in healthy older adults: effects of postural task difficulty and cognitive load. Gait Posture 2011;33(4):651-5.

22. Mazo GZ, Mota J, Benedetti TB, De Barros MVG. Validade concorrente e reprodutibilidade: teste-reteste do Questionário de Baecke modificado para idosos. Rev Bras AtivFís Saúde 2001;6(1):5-11.

23. Paulus WM, Straube A, Brandt T. Visual stabilization of posture: physiological stimulus characteristics and clinical aspects. Brain 1984;107 (4):1143-63.

24. Wayne PM, Kiel DP, Buring JE, Connors EM, Bonato P, Yeh GY, et al. Impact of Tai Chi exercise on multiple fracture-related risk factors in post-menopausal osteopenic women: a pilot pragmatic, randomized trial. BMC Complement Altern Med 2012;12:7.

25. Freiberger E, Haberle L, Spirduso WW, Zijlstra GA. Long-term effects of three multicomponent exercise interventions on physical performance and fall-related psychological outcomes in community-dwelling older adults: a randomized controlled trial. J Am Geriatr Soc 2012;60(3):437-46.

26. Gupta SK. Intention-to-treat concept: a review. Perspect Clin Res 2011;2(3):109-12.

27. Shrier I, Steele RJ, Verhagen E, Herbert R, Riddell CA, Kaufman JS. Beyond intention to treat: what is the right question? Clin Trials 2014;11(1):28-37.

28. Rubenstein LZ, Josephson KR, Trueblood PR, Loy S, Harker JO, Pietruska FM,et al. Effects of a group exercise program on strength, mobility, and falls among fall-prone elderly men. J Gerontol Ser A Biol Sci Med Sci 2000;55(6):317-21.

29. Shimada H, Obuchi S, Furuna T, Suzuki T. New intervention program for preventing fall among frail elderly people: the effects of perturbed walking exercise using a bilateral separated treadmill. Am J Phys Med Rehabilit 2004;83(7):493-9.

30. Gillespie LD, Robertson MC, Gillespie WJ, Lamb SE, Gates S, Cumming RG, et al.. Interventions for preventing falls in older people living in the community. Cochrane Database Syst Rev 2009;15(2):1-4.. 
31. Brouwer BJ, Walker C, Rydahl SJ, Culham EG. Reducing fear of falling in seniors through education and activity programs: a randomized trial. J Am Geriatr Soc 2003;51(6):829-34.

32. Jessup JV, Horne C, Vishen RK, Wheeler D. Effects of exercise on bone density, balance, and self-efficacy in older women. Biol Res Nurs 2003;4(3):171-80.

33. Lord SR, Tiedemann A, Chapman K, Munro B, Murray SM;Gerontology M, et al. The effect of an individualized fall prevention program on fall risk and falls in older people: a randomized, controlled trial. J Am Geriatr Soc 2005;53(8):1296-304.

34. Ramsbottom R, Ambler A, Potter J, Jordan B, Nevill A, Williams C. The effect of 6 months training on leg power, balance, and functional mobility of independently living adults over 70 years old. J Aging Phys Act 2004;12(4):497-510.

35. Judge JO, Lindsey C, Underwood M, Winsemius D. Balance improvements in older women: effects of exercise training. Phys Ther 1993;73(4):254-62.

Received: March 2, 2015

Reviewed: November 25, 2015

Accepted: April 11, 2016 